\section{FRI0591 VALIDITY OF THE GERMAN VERSION OF BOTH THE PARENT ADHERENCE REPORT QUESTIONNAIRE (PARC) AND THE CHILD ADHERENCE REPORT QUESTIONNAIRE (CARQ) - DATA OF THE INCEPTION COHORT OF NEWLY DIAGNOSED PATIENTS WITH JUVENILE IDIOPATHIC ARTHRITIS (ICON)}

S. Kirchner ${ }^{1}$, C. Sengler ${ }^{1}$, J. Klotsche ${ }^{1}$, I. Liedmann ${ }^{1}$, M. Niewerth ${ }^{1}$,

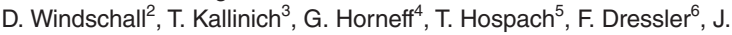
B. Kuemmerle-Deschner ${ }^{7}$, K. Minden ${ }^{1} .{ }^{1}$ German Rheumatism Research Center, Berlin, Germany; ${ }^{2}$ St-Josef-Stitt, Sendenhorst, Germany; ${ }^{3}$ Charité - Universitätsmedizin Berlin, Department of Pediatrics, Berlin, Germany; ${ }^{4}$ Asklepios Klinik St. Augustin, St. Augustin, Germany; ${ }^{5}$ Olgahospital, Department of Pediatrics, Stuttgart, Germany; ${ }^{6}$ Children's Hospital, Medical School, Hannover, Germany; ${ }^{7}$ University Children's hospital, Tübingen, Germany

Background: Juvenile idiopathic arthritis (JIA) is the most common chronic inflammatory rheumatic disease in childhood. A multimodal treatment is needed to reduce pain, control inflammation and maintain joint functioning. Adherence to prescribed therapies is necessary for an optimal outcome. Measuring adherence in children with JIA and their caregivers by a validated questionnaire provides important information about benefits and problems with treatment.

Objectives: To evaluate adherence in JIA patients and to validate the German version of both the parent adherence report questionnaire (PARQ) and the child adherence report questionnaire (CARQ).

Methods: The PARQ and CARQ were translated from its original English version into German and cross-culturally adapted. Parents and children completed the PARQ and CARQ 4 years after enrolment in the Inception cohort ICON. These questionnaires measure child ability (by VAS 0-100, $100=$ best) related to i) general level of difficulty in following treatment, ii) frequency of following treatment, iii) negative reactions in response to treatment [i)-iii) summarized to child ability total score], iv) perceived helpfulness of treatment, and 4 categorical questions on errors in medication behavior. Reliability was tested by re-administering the questionnaire after a mean of 13 days. Reproducibility was analysed using intraclass correlation coefficients (ICC). VAS scores were correlated with the Pediatric Quality of Life Inventory (PedsQL) treatment scale items for convergent validity, and with sociodemographic parameters for discriminant validity.

Results: 481 parents and 465 children completed the PARQ and the CARQ, respectively, 56 parents and 37 children took part in the re-test. The mean age at assessment was $10.1 \pm 3.7$ years, mean disease duration was $4.7 \pm 0.8$ years. The majority of patients suffered from oligoarthritis (49\%), followed by rheumatoid-factor negative polyarthritis (30\%). Treatment with a DMARD received $60 \%$ (MTX 46\%), 28\% received a biological drug, $16 \%$ both. Disease activity measured by the clinical juvenile arthritis disease activity score-10 (cJADAS-10) was $2.6 \pm 3.4$ (range $0-30$, best $=0$ ), functional status was good (mean $\mathrm{CHAQ}$ $0.2 \pm 0.4$ ). Exercise and splints were prescribed to $57 \%$ and $21 \%$ of patients, respectively.

PARQ/CARQ mean child ability total scores for medication were $73.1 \pm$ $23.3 / 76.5 \pm 24.2$, for exercise: $85.6 \pm 16.5 / 90.3 \pm 15.0$, for splints: $72.9 \pm$ $24.2 / 82.9 \pm 16.5$. About a third of parents and children reported any error in medication behavior. Perceived helpfulness was highest for medication (PARQ/ CARQ 87.4 $\pm 20.6 / 83.6 \pm 26.1$ ) and lowest for splints. (PARQ/CARQ $80.8 \pm$ $28.4 / 73.5 \pm 33.6$.

ICCs related to medication indicated good to excellent concordance (PARQ ICC $=$ 0.69 - 0.96; CARQ ICC $=0.53-0.75)$, to exercise moderate $(P A R Q$ ICC $=0.28$ 0.45 ; CARQ ICC $=0.67-0.93$ ) and to splints disparate concordance (PARQ ICC $=0.01-0.90$, CARQ ICC $=0.86-0.93$ )

Scores for medications (PARQ: 0.06 - 0.38, CARQ: 0.06 - 0.49), exercise (PARQ: r 0.03 - 0.30, CARQ: $0.01-0.34$ ) and splints (PARQ: r 0.09 - 0.52, CARQ: 0.11 - 0.62) showed a fair to good correlation with the PedsQL scales. Gender and socioeconomic status were not associated with the level of adherence.

Conclusion: The German version of the PARQ and CARQ appears to be a valuable tool to measure adherence in patients with JIA and to evaluate helpfulness of treatments.

Acknowledgments: ICON is funded by the Federal Ministry of Research (FKZ:01ER0812)

Disclosure of Interests: Sabine Kirchner: None declared, Claudia Sengler: None declared, Jens Klotsche: None declared, Ina Liedmann: None declared, Martina Niewerth: None declared, Daniel Windschall: None declared, Tilmann Kallinich Grant/research support from: Novartis, Consultant of: Sobi, Roche, Novartis, Gerd Horneff Grant/research support from: AbbVie, Chugai, Merck Sharp \& Dohme, Novartis, Pfizer, Roche, Speakers bureau: AbbVie, Bayer, Chugai, Merck Sharp \& Dohme, Novartis, Pfizer, Roche, Toni Hospach: None declared, Frank Dressler: None declared, J. B. Kuemmerle-Deschner Grant/ research support from: Novartis, AbbVie, Sobi, Consultant of: Novartis, AbbVie, Sobi, Kirsten Minden Consultant of: GlaxoSmithKline, Sanofi, Speakers bureau: Roche

DOI: 10.1136/annrheumdis-2020-eular.4215

\section{FRI0592 \\ IMPACT OF INDIVIDUAL SYMPTOMS OF PSORIATIC ARTHRITIS ON PHYSICAL COMPONENT SCORE AND MENTAL COMPONENT SCORE OF SF-36 AS A MEASURE OF HEALTH RELATED QUALITY OF LIFE (QOL): AN OBSERVATIONAL COHORT STUDY}

M. Skougaard ${ }^{1}$, T. Schjødt Jørgensen ${ }^{1}$, M. J. Jensen ${ }^{1}$, C. Ballegaard ${ }^{1}$, J. GuldbergMøller ${ }^{1}$, A. Egeberg ${ }^{2}$, R. Christensen', J. F. Merola ${ }^{3}$, L. C. Coates ${ }^{4}$, V. Strand ${ }^{5}$, P. J. Mease ${ }^{6}$, L. E. Kristensen ${ }^{1}{ }^{1}$ The Parker Institute, Bispebjerg and Frederiksberg Hospital, Copenhagen, Denmark; ${ }^{2}$ Department of Dermatology and Allergy, Herlev and Gentofte Hospital, Copenhagen, Denmark; ${ }^{3}$ Department of Dermatology and Department of Medicine, Division of Rheumatology, Brigham and Women's Hospital, Harvard Medical School, Boston, United States of America; ${ }^{4}$ Nuffield Department of Orthopaedics, Rheumatology and Musculoskeletal Sciences, University of Oxford, Oxford, United Kingdom; ${ }^{5}$ Division Immunology/ Rheumatology, Stanford University, Palo Alto, United States of America; ${ }^{6}$ Swedish Medical Centre, and University of Washington, Seattle, United States of America

Background: Patients with Psoriatic Arthritis (PsA) experience diverse symptoms including skin and nail psoriasis, swollen and tender joints, enthesitis, and fatigue that have shown to impair health related quality of life (QoL). We hypothesized that different elements of disease influence SF-36 physical (PCS) and menta (MCS) component summary scores differently.

Objectives: The objective of the study was to assess the interaction between change in disease activity (DAS28CRP), PsA symptoms (psoriasis [PsO], nail $\mathrm{PsO}$, enthesitis, fatigue, pain, and physical function) with changes in PCS and MCS scores in a PsA patient cohort exploring effect of treatment on clinical manifestations and patient-reported outcome (PRO).

Methods: Data were obtained from the PIPA cohort (1) at baseline and after 4 months of treatment. Patients' characteristics were described as medians with interquartile ranges (IQRs) and numbers with percentages. Data were presented as

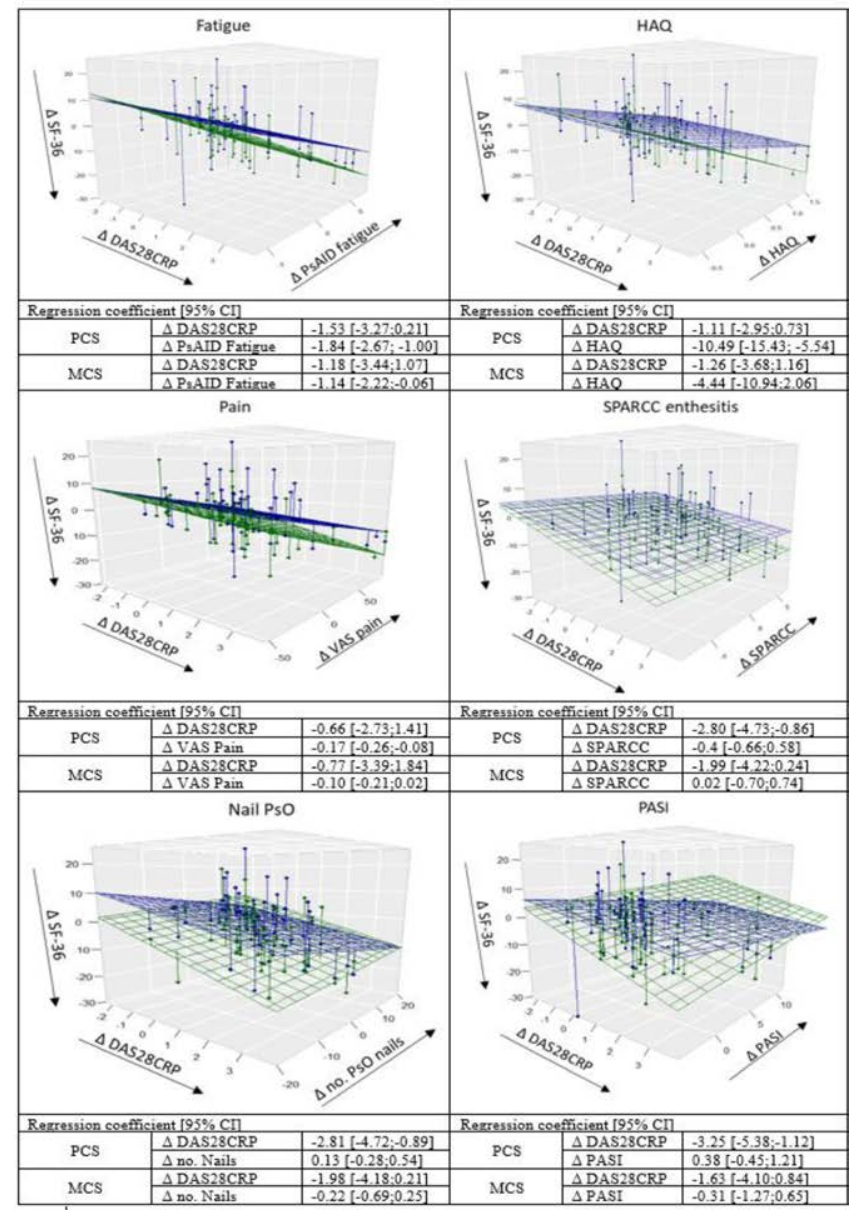

Figure 1. Association between disease activity, individual symptoms and PCS/MCSPCS; physical component summary (green regression plane), MCS; mental component summary (blue regression plane). Arrows indicate the positive improvement vector. SF-36: short form-36, Cl: Confidence Interval, DAS28CRP: disease activity score with 28 joints and c-reactive protein PASI: Psoriasis Area Severity Index, SPARCC: Spondyloarthritis Research Consortium of Canada enthesitis index, VAS: visual analogue scale, PsAID: Psoriatic Arthritis Impact of Disease, $\mathrm{HAQ}$ : Health Assessment Questionnaire 
changes between baseline and follow-up with delta $(\Delta)$ values on xyz-plots. Associations between PCS and MCS scores, DAS28CRP, and PsA symptoms were described with fitted linear regression plane models. PCS and MCS were derived from 8 domains of SF-36 and ranged from 0-100 with lower values reflecting more impaired QoL.

Results: 71 PsA patients were included in the study. 40 (56\%) patients were female with a mean age of 50 (IQR 41-60) years and disease duration of 2.15 (IQR 0.2-9) years. Figure 1 shows associations between PsA symptoms, DAS28CRP, and PCS (green regression plane) and MCS (blue regression plane). For all PROs; pain, fatigue and physical function, improvements in both $\triangle \mathrm{PCS}$ and $\triangle$ MCS scores were associated with improvements in either $\triangle$ pain, $\triangle P$ SAID fatigue, and/or $\triangle \mathrm{HAQ}$, and to a larger extent than improvements in $\triangle \mathrm{DAS} 28 C R P$. Improvements in $\triangle$ nail PsO (regression coefficient (RC): -0.22) and $\triangle P A S I$ (RC: -0.31) positively impacts $\triangle \mathrm{MCS}$, without a clear association in PCS scores (RC: 0.13 and 0.38 for $\Delta$ nail $\mathrm{PsO}$ and $\triangle \mathrm{PASI}$, respectively). Improvement in inflammatory features SPARCC enthesitis and DAS28CRP showed improvement in both $\triangle \mathrm{PCS}$ and $\triangle \mathrm{MCS}$.

Conclusion: Pain and fatigue are well-known factors to impair QoL in PsA patient. Here we show that diminishing these factors, pain and fatigue, improved both PCS and MCS scores more than changes in DAS28CRP. Improvements in skin and nail manifestations impacted MCS scores and are as important as changes in joint manifestations which affect PCS and MCS scores equally.

\section{References:}

[1] Hojgaard $P$ et al. Pain mechanisms and ultrasonic inflammatory activity as prognostic factors in patients with psoriatic arthritis (...) BMJ Open. 20

Disclosure of Interests: Marie Skougaard: None declared, Tanja Schjødt Jørgensen Speakers bureau: Abbvie, Pfizer, Roche, Novartis, UCB, Biogen, and Eli Lilly, Mia Joranger Jensen: None declared, Christine Ballegaard: None declared, Jørgen Guldberg-Møller Speakers bureau: Novartis, Ely Lilly, AbbVie, BK Ultrasound, Alexander Egeberg Grant/research support from: Pfizer, Eli Lilly, Novartis, AbbVie, Janssen Pharmaceuticals, the Danish National Psoriasis Foundation and the Kgl Hofbundtmager Aage Bang Foundation, Consultant of: UCB Pharma (Advisory Board), Speakers bureau: AbbVie, Almirall, Leo Pharma, Samsung Bioepis Co. Ltd., Pfizer, Eli Lilly, Novartis, Galderma, Dermavant, UCB Pharma, Mylan, Bristol-Myers Squibb and Janssen Pharmaceuticals, Robin Christensen: None declared, Joseph F. Merola Consultant of: Merck, AbbVie, Dermavant, Eli Lilly, Novartis, Janssen, UCB Pharma, Celgene, Sanofi, Regeneron, Arena, Sun Pharma, Biogen, Pfizer, EMD Sorono, Avotres and LEO Pharma, Laura C Coates: None declared, Vibeke Strand Consultant of: AbbVie, Amgen, Biogen, Celltrion, Consortium of Rheumatology Researchers of North America, Crescendo Bioscience, Eli Lilly, Genentech/ Roche, GlaxoSmithKline, Hospira, Janssen, Merck, Novartis, Pfizer, Regeneron Pharmaceuticals, Inc., Sanofi, UCB, Philip J Mease Grant/research support from: Abbott, Amgen, Biogen Idec, BMS, Celgene Corporation, Eli Lilly, Novartis, Pfizer, Sun Pharmaceutical, UCB - grant/research support, Consultant of: Abbott, Amgen, Biogen Idec, BMS, Celgene Corporation, Eli Lilly, Novartis, Pfizer, Sun Pharmaceutical, UCB - consultant, Speakers bureau: Abbott, Amgen, Biogen Idec, BMS, Eli Lilly, Genentech, Janssen, Pfizer, UCB - speakers bureau, Lars Erik Kristensen Consultant of: UCB Pharma (Advisory Board), Sannofi (Advisory Board), Abbvie (Advisory Board), Biogen (Advisory Board), Speakers bureau: AbbVie, Amgen, Biogen, Bristol-Myers Squibb,Celgene, Eli Lilly, Gilead, Forward Pharma, Janssen Pharmaceuticals, MSD, Novartis, Pfizer, and UCB Pharma

DOI: 10.1136/annrheumdis-2020-eular.4071

\section{FRI0593 CORRELATION BETWEEN DISEASE ACTIVITY AND MENTAL HEALTH OF AS PATIENTS: A CROSS- SECTION STUDY WITH SELF-ASSESSMENTS BASED ON SMART SYSTEM OF DISEASE MANAGEMENT (SSDM) MOBILE TOOLS}

H. Song ${ }^{1}$, H. Wei ${ }^{2}$, M. Zhang ${ }^{3}$, L. Wu ${ }^{4}$, Z. Wu ${ }^{5}$, C. Aichun ${ }^{6}$, B. Wang ${ }^{7}$, X. Wang ${ }^{8}$,

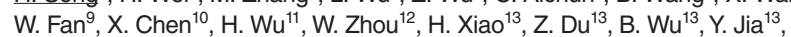
F. Xiao ${ }^{13}$, J. Lu ${ }^{14}$ on behalf of SSDM Collaboration Group, China. ${ }^{1}$ Beijing Jishuitan Hospital, Beijing, China; ${ }^{2}$ Northern Jiangsu People's Hospital, Yangzhou, China; ${ }^{3}$ Jiangsu Province Hospital, The first affiliated hospital of Nanjing medical university, Nanjing, China; ${ }^{4}$ People's Hospital of Xinjiang Uygur Autonomous Region, Urumqi, China; ${ }^{5}$ The First Affiliated Hospital of The Air Force Medical University, Xi'an, China; ${ }^{6}$ Renmin Hospital of Wuhan University Hubei General Hopital, Wuhan, China; ${ }^{7}$ Beijing Hospital of Traditional Chinese Medicine, Beijing, China; ${ }^{8}$ Anyang district hospital, Anyang, China; ${ }^{9}$ Central Hospital of XinXiang, Henan, China; ${ }^{10}$ Zhongnan Hospital of Wuhan University, Wuhan, China; ${ }^{11}$ Dongguan Donghua Hospital, Dongguan, China; ${ }^{12}$ Wuhan No. 4 Hospital, Wuhan, China; ${ }^{13}$ Shanghai Gothic Internet Technology Co., Ltd., Shanghai, China; ${ }^{14}$ First Affiliated Hospital of China Medical University, Shengyang, China
Background: WHO survey showed that the prevalence of anxiety and depression in Chinese population and Chinese patients with chronic diseases were between $3.1 \%-4.2 \%$ and $3.1 \%-7.3 \%$, respectively. Ankylosing Spondylitis Disease Activity Score (ASDAS) and Hospital Anxiety and Depression Scale (HADS) are commonly used to evaluate AS patients' disease activity and mental health. All those assessments were mainly performed by health professionals (HCPs) with paper questionnaire previously. SSDM is a novel smart disease management tool that allows patients to do self-assessments on ASDAS and HADS by mobile terminals. Objectives: To estimate the prevalence of anxiety and depression in Chinese patients with AS and to analyze the potential association between disease activity and mental health.

Methods: Under the guidance and training by HCPs, AS patients downloaded SSDM and performed self-assessments bundle of ASDAS and HADS with SSDM. ASDAS $<=1.3,1.3-2.1,2.1-3.5$ and $>3.5$ are defined as inactive (IDA), moderate (MDA), high (HDA) and very high (VHDA) disease activity, respectively. ASDAS score $<=1.3$ represents inactive disease status and achievement of T2T. HADS score $>=8$ can be diagnosed with anxiety or depression.

Results: From June 2016 to Jan 2020, 1,931 AS patients (1,118 male, 813 female) with a mean age of $34.09 \pm 11.86(12-82)$ years and the median disease duration of 2.61 years from 207 hospitals performed bundle self-assessments for 2,477 times in total. According to the HADS and ASDAS assessment results, the prevalence of anxiety and depression in all patients was $36.7 \%$ and $39.3 \%$ respectively, which was significantly higher than that in the WHO survey in Chinese population and chronic disease patients. The proportion of patients achieved and failed on T2T was $29 \%$ and $71 \%$, respectively. The prevalence of anxiety (A) and depression (D) was $25 \%$ and $23 \%$ among T2T achievers; and $37 \%$ and $32 \%$ among T2T failures, respectively ( $\mathrm{pA}<0.05, \mathrm{pD}<0.05)$.

According to ASDAS, in IDA, MDA, HDA and VHDA subgroups, the prevalence of anx iety and depression was $27 \%, 36 \%, 41 \%, 52 \%$ and $29 \%, 38 \%, 45 \%, 56 \%$, respectively. The correlation coefficients of anxiety (A) and depression (D) with ASDAS were $r A=0.9908$ and $r D=0.9964$. It suggested that with the increase of disease activity, the proportion of AS patients with anxiety and depression increased significantly. (Figure 1) Conclusion: The prevalence of anxiety and depression in AS patients was significantly higher than that in the WHO survey in Chinese population and chronic disease patients. Higher prevalence of anxiety and depression were associated with higher levels of disease activity. SSDM is an effective mobile interface to monitor and study entanglement of disease activity and mental health in AS patients, which build a foundation for proactive interventions in future.

The prevalence of anxiety and depression according to ASDAS

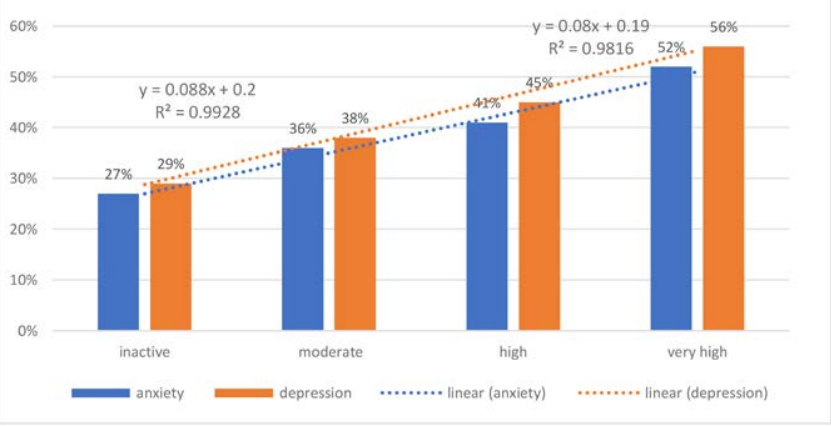

Figure 1. The prevalence of anxiety and depression according to ASDAS.

Acknowledgments: Smart system of disease management (SSDM) was developed by Shanghai Gothic Internet Technology Co., Ltd.

Disclosure of Interests: None declared

DOI: 10.1136/annrheumdis-2020-eular.1774

\section{FRI0594 \\ USE OF ANTI-DFS70 ANTIBODIES IN RHEUMATOLOGICAL PATIENTS WITH SUSPICION OF SYSTEMIC AUTOIMMUNE DISEASE}

J. L. Tandaipan Jaime ${ }^{1}$, B. Magallares ${ }^{2}$, J. Bernardez¹ , E. Riera Alonso, F. Pujalte ${ }^{3}$, L. Martínez-Martínez ${ }^{4}$, A. Baucells ${ }^{4}$, I. Castellví ${ }^{2}$, H. Corominas ${ }^{2}$, S. Martinez Pardo'. ' ${ }^{1}$ Hospital Universitari Mutua Terrassa, Rheumatology, Terrassa, Spain; ${ }^{2}$ Hospital de la Santa Creu I Sant Pau, Rheumatology, Barcelona, Spain; ${ }^{3}$ Catlab, Inmunology, Terrassa, Spain; ${ }^{4}$ Hospital de la Santa Creu I Sant Pau, Inmunology, Barcelona, Spain

Background: Positivity of dense fine speckles antibodies (anti-DFS70) has been associated with antinuclear antibodies positivity in people with no evidence of 